\title{
Existence and stability of superconducting solutions for the Ginzburg-Landau equations in the presence of weak electric currents
}

\author{
Yaniv Almog; Leonid Berlyand† Dmitry Golovaty; and Itai Shafrir ${ }^{\S}$
}

\begin{abstract}
For a reduced Ginzburg-Landau model in which the magnetic field is neglected, we prove, for weak electric currents, the existence of a steady-state solution in a vicinity of the purely superconducting state. We further show that this solution is linearly stable.
\end{abstract}

\section{Introduction}

Superconducting materials are characterized by a complete loss of resistivity at temperatures below some critical threshold value. In this state, electrical current can flow through a superconducting sample while generating only a vanishingly small voltage drop. If the current is increased above a certain critical level, however, superconductivity is destroyed and the material reverts to the normal state even while it remains below the critical temperature.

In this work, we study this phenomenon within the framework of the timedependent Ginzburg-Landau model [1, 2], presented here in a dimensionless form

$$
\left\{\begin{array}{cl}
\frac{\partial u}{\partial t}+i \phi u=(\nabla-i A)^{2} u+u\left(1-|u|^{2}\right) & \text { in } \Omega \times \mathbb{R}_{+}, \\
-\kappa^{2} \nabla \times \nabla \times A+\sigma\left(\frac{\partial A}{\partial t}+\nabla \phi\right)=\Im\{\bar{u} \nabla u\}+|u|^{2} A & \text { in } \Omega \times \mathbb{R}_{+}, \\
(i \nabla+A) u \cdot \nu=0 \quad \text { and } \quad-\sigma\left(\frac{\partial A}{\partial t}+\nabla \phi\right) \cdot \nu=J & \text { on } \partial \Omega \times \mathbb{R}_{+}, \\
u(x, 0)=u_{0} \quad \text { and } \quad A(x, 0)=A_{0} & \text { in } \Omega,
\end{array}\right.
$$

In the above system of equations, $u$ is the order parameter with $|u|$ representing the number density of superconducting electrons. Materials for which $|u|=1$ are said to be purely superconducting while those for which $u=0$ are said to be in

*Department of Mathematics, Louisiana State University, Baton Rouge, LA 70803, USA

${ }^{\dagger}$ Department of Mathematics, Pennsylvania State University, University Park, PA 16802, USA

${ }^{\ddagger}$ Department of Mathematics, The University of Akron, Akron, Ohio 44325, USA

${ }^{\S}$ Department of Mathematics, Technion - Israel Institute of Technology, 32000 Haifa, Israel 
the normal state. We denote the magnetic vector potential by $A$-so that the magnetic field is given by $h=\nabla \times A$ - and by $\phi$ the electric scalar potential. The constants $\kappa$ and $\sigma$ are the Ginzburg-Landau parameter and normal conductivity, of the superconducting material, respectively, and the quantity $-\sigma\left(A_{t}+\nabla \phi\right)$ is the normal current. All lengths in (1) have been scaled with respect to the coherence length $\xi$ that characterizes spatial variations in $u$. The domain $\Omega \subset \mathbb{R}^{2}$ occupied by the superconducting sample is separated from its exterior by the boundary $\partial \Omega$ that consists of two parts, $\partial \Omega=\partial \Omega_{c} \cup \partial \Omega_{i}$. Here $\partial \Omega_{c}$ corresponds to the portion of the boundary through which current enters and exits the sample, while the rest of the boundary, denoted by $\partial \Omega_{i}$, is electrically insulated. The function $J: \partial \Omega \rightarrow \mathbb{R}$ with $\operatorname{supp}(J)=\partial \Omega_{c}$ represents the normal current entering the sample. Note, that it is possible to prescribe the electric potential on $\partial \Omega$ instead of the current. [1]

Except for the initial conditions, (11) is invariant under the gauge transformation

$$
A \rightarrow A+\nabla \omega \quad ; \quad u \rightarrow u e^{i \omega} \quad ; \quad \phi \rightarrow \phi-\frac{\partial \omega}{\partial t}
$$

for some smooth $\omega$. Finally, one has to prescribe $h$ at a single point on $\partial \Omega$ for all $t>0$ (cf. [3]).

It has been demonstrated in [3], for a fixed current, that in the limit $\kappa \rightarrow \infty$ one can formally obtain from (11) the following system of equations

$$
\begin{cases}\frac{\partial u}{\partial t}+i \phi u=\Delta u+u\left(1-|u|^{2}\right) & \text { in } \Omega \times \mathbb{R}_{+}, \\ \sigma \Delta \phi=\nabla \cdot[\Im(\bar{u} \nabla u)] & \text { in } \Omega \times \mathbb{R}_{+}, \\ \frac{\partial u}{\partial \nu}=0 \text { and }-\sigma \frac{\partial \phi}{\partial \nu}=J & \text { on } \partial \Omega \times \mathbb{R}_{+}, \\ u(x, 0)=u_{0} & \text { in } \Omega .\end{cases}
$$

The principal goal of the present paper is to study (2) in the large domain limit. To this end, we apply the transformation

$$
t^{1}=\epsilon^{2} t ; x^{1}=\epsilon x ; J^{1}=\frac{J}{\epsilon} ; \phi^{1}=\frac{\phi}{\epsilon^{2}} ; \sigma^{1}=\sigma \epsilon^{2},
$$

to (2) and drop the superscript 1 for notational convenience to obtain

$$
\begin{cases}\frac{\partial u}{\partial t}+\mathcal{L}_{\epsilon} u=0 & \text { in } \Omega \times \mathbb{R}_{+}, \\ \sigma \Delta \phi=\nabla \cdot[\Im(\bar{u} \nabla u)] & \text { in } \Omega \times \mathbb{R}_{+}, \\ \frac{\partial u}{\partial \nu}=0 \text { and }-\sigma \frac{\partial \phi}{\partial \nu}=J & \text { on } \partial \Omega \times \mathbb{R}_{+}, \\ u(x, 0)=u_{0} & \text { in } \Omega .\end{cases}
$$

Here

$$
\mathcal{L}_{\epsilon} u=i \phi u-\Delta u-\frac{u}{\epsilon^{2}}\left(1-|u|^{2}\right) .
$$

We assume in the sequel that $\Omega$ in (3) is independent of $\epsilon$. 
Note that (3) remains invariant under the transformation

$$
u \rightarrow e^{i \omega(t)} u \quad ; \quad \phi \rightarrow \phi+\frac{\partial \omega}{\partial t}
$$

We thus choose

$$
\omega=-\int_{0}^{t} \frac{\left(|u|^{2} \phi\right)_{\Omega}(\tau)}{\left(|u|^{2}\right)_{\Omega}(\tau)} d \tau
$$

which guarantees that we have for all $t>0$,

$$
\left(|u|^{2} \phi\right)_{\Omega}(t) \equiv 0
$$

where

$$
(f)_{\Omega}:=\frac{1}{|\Omega|} \int_{\Omega} f d x
$$

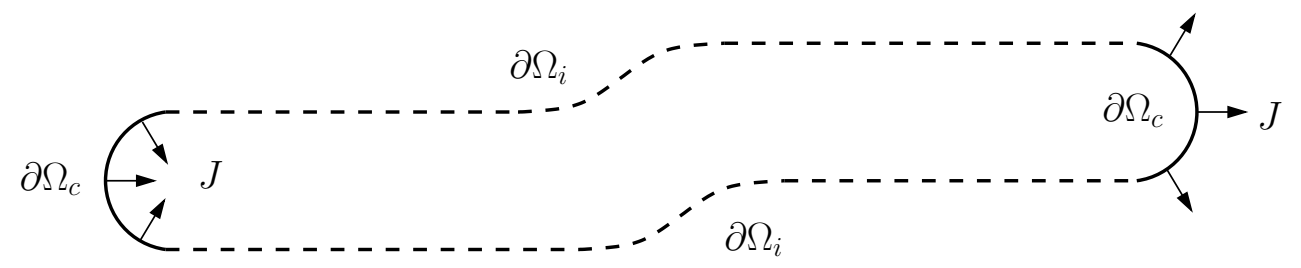

Figure 1: Schematics of a superconducting sample. The arrows denote the direction of the current flow.

The system of equations (11) for a variety of domains and boundary conditions has attracted significant interest among both physicists [4]-8] and mathematicians [3], 9]-[13]. A different simplification of (3) was derived by Du \& Gray [14] for the same limit $(\kappa \rightarrow \infty)$, but assuming that $J$ and $\sigma$ are of order $\mathcal{O}\left(\kappa^{2}\right)$ (cf. [15]).

The focus of this work is mainly on the existence and stability of steady-state solutions of (3) for relatively small currents. The main result that we prove is the following

Theorem 1. Let $0<\delta_{0}$ and suppose that $\epsilon\|J\|_{H^{3 / 2}(\partial \Omega)} \leq \delta_{0}$. Then

1. The system (3) possesses a steady-state solution $\left(u_{s}, \phi_{s}\right) \in H^{2}(\Omega, \mathbb{C}) \times H^{2}(\Omega, \mathbb{R})$ whenever $\delta$ is sufficiently small. Furthermore, there exists a constant $C(\Omega, \sigma)$, independent of both $\epsilon$ and $\delta_{0}$, such that

$$
\left\|1-\left|u_{s}\right|\right\|_{2,2} \leq C \delta^{2} .
$$

2. The solution $\left(u_{s}, \phi_{s}\right)$ is linearly stable in the following sense. Given

$$
\mathcal{U}=\left\{u \in H^{2}(\Omega, \mathbb{C}): \partial u /\left.\partial \nu\right|_{\partial \Omega}=0\right\},
$$


let $\mathcal{L}_{\epsilon}: \mathcal{U} \rightarrow L^{2}(\Omega, \mathbb{C})$ be defined by (4) where the potential $\phi(u)$ is assumed to solve

$$
\begin{cases}\sigma \Delta \phi=\nabla \cdot[\Im(\bar{u} \nabla u)] & \text { in } \Omega \times \mathbb{R}_{+}, \\ -\sigma \frac{\partial \phi}{\partial \nu}=J & \text { on } \partial \Omega \times \mathbb{R}_{+}, \\ \left(|u|^{2} \phi\right)_{\Omega}(t) \equiv 0 . & \end{cases}
$$

Then, there exist a $0 \leq \delta_{1} \leq \delta$ such that, whenever $\epsilon\|J\|_{H^{3 / 2}(\partial \Omega)} \leq \delta_{1}$, the semi-group associated with the Fréchet derivative $D \mathcal{L}_{\epsilon}\left(u_{s}\right)$ is asymptotically stable.

Remark 1. Note that Theorem 1 is valid for every $0<\epsilon \leq 1$ and in particular when $\epsilon \ll \delta$.

The rest of this paper is organized as follows. In the next section, we prove the existence of steady-state solutions and discuss their properties in Theorem 2. The stability of these solutions (Proposition 2) is subsequently demonstrated in Section 3 .

\section{$2 \quad$ Steady state solutions}

In this section, we consider the steady-state solutions of (3). Let $(u, \phi)$ denote a smooth solution of

$$
\left\{\begin{array}{cc}
-\Delta u+i \phi u=\frac{u}{\epsilon^{2}}\left(1-|u|^{2}\right) & \text { in } \Omega, \\
\sigma \Delta \phi=\nabla \cdot[\Im(\bar{u} \nabla u)] & \text { in } \Omega, \\
\frac{\partial u}{\partial \nu}=0 \text { and }-\sigma \frac{\partial \phi}{\partial \nu}=J & \text { on } \partial \Omega
\end{array}\right.
$$

If we set $u=\rho e^{i \chi}$ the problem takes the form

$$
\left\{\begin{array}{cc}
-\Delta \rho+\rho|\nabla \chi|^{2}=\frac{\rho}{\epsilon^{2}}\left(1-\rho^{2}\right) & \text { in } \Omega, \\
\operatorname{div}\left(\rho^{2} \nabla \chi\right)=\rho^{2} \phi & \text { in } \Omega, \\
\sigma \Delta \phi=\operatorname{div}\left(\rho^{2} \nabla \chi\right) & \text { in } \Omega, \\
\frac{\partial \rho}{\partial \nu}=\frac{\partial \chi}{\partial \nu}=0 \text { and }-\sigma \frac{\partial \phi}{\partial \nu}=J & \text { on } \partial \Omega, \\
(\chi)_{\Omega}=0 . &
\end{array}\right.
$$

In what follows, we assume that $J$ belongs to $H^{3 / 2}(\partial \Omega)$ and set $\|J\|$ to be the $H^{3 / 2}$ norm of $J$. Note that (7) is imposed in order to eliminate the degree of freedom that results from the invariance of (7 $\mathrm{a}-\mathrm{e})$ with respect to the transformation $\chi \rightarrow \chi+C$, for any constant $C$. Moreover, any solution of (7) must satisfy

$$
\int_{\Omega} \rho^{2} \phi=0
$$


as can be easily verified by integrating (17b) and then using (77,e).

Assuming that current is sufficiently small, we seek an approximation to the solution of (7) that would be uniform in $\epsilon$ and, in particular, would remain valid in the limit $\epsilon \rightarrow 0$. To this end, we fix the value of $\sigma$ while allowing for some dependence of $J$ on $\epsilon$ : a point that will be clarified in the sequel. The approximate solution $(u, \phi)=\left(\rho_{0} e^{i \chi_{0}}, \phi_{0}\right)$ when $\epsilon \ll 1$ should satisfy

$$
\begin{cases}\rho_{0}^{2}=1-\epsilon^{2}\left|\nabla \chi_{0}\right|^{2} & \text { in } \Omega, \\ -\sigma \Delta \phi_{0}+\rho_{0}^{2} \phi_{0}=0 & \text { in } \Omega, \\ \operatorname{div}\left(\rho_{0}^{2} \nabla \chi_{0}\right)=\rho_{0}^{2} \phi_{0} & \text { in } \Omega, \\ \frac{\partial \phi_{0}}{\partial \nu}=-\frac{J}{\sigma} & \text { on } \partial \Omega_{c}, \\ \frac{\partial \chi_{0}}{\partial \nu}=0 & \text { on } \partial \Omega_{i}, \\ \left(\chi_{0}\right)_{\Omega}=0 . & \end{cases}
$$

Note that the only term dropped from (7) to obtain (9) is $-\Delta \rho$ in (7a). We first prove the existence of solutions to (9) for a sufficiently small

$$
\delta:=\|J\| \epsilon .
$$

As will become clear later on, the solution of (9), whose existence is proved below, serves a good approximation for a solution of (7) whenver $\delta$ is sufficiently small, even if $\epsilon$ is bounded away from zero.

Proposition 1. Let

$$
\mathcal{H}_{1}=\left\{\phi \in H^{3}(\Omega)|\partial \phi / \partial \nu|_{\partial \Omega}=0\right\} ; \mathcal{H}_{2}=\left\{\chi \in H^{3}(\Omega)|\partial \chi / \partial \nu|_{\partial \Omega}=0,(\chi)_{\Omega}=0\right\},
$$

and let $\mathcal{W}_{1}=\mathcal{H}_{1} \times \mathcal{H}_{2}$. There exist positive $\delta_{0}$ and $C(\Omega, \sigma)$, such that the problem (9) possesses a solution $\left(\chi_{0}, \phi_{0}\right) \in \mathcal{W}_{1}$ satisfying

$$
\left\|\chi_{0}\right\|_{3,2}+\left\|\phi_{0}\right\|_{3,2} \leq C\|J\|
$$

and

$$
\left\|\left(1-\rho_{0}\right)\right\|_{2,2} \leq C \delta^{2}
$$

for all $0<\delta<\delta_{0}$ and $\sigma>0$.

Proof. We make use of the implicit function theorem to prove the proposition. Denote by $\phi_{0,0}$ and $\chi_{0,0}$ the solutions of

$$
\begin{cases}-\sigma \Delta \phi_{0,0}+\phi_{0,0}=0 & \text { in } \Omega, \\ \frac{\partial \phi_{0,0}}{\partial \nu}=-\frac{J}{\sigma} & \text { on } \partial \Omega,\end{cases}
$$


and

$$
\begin{cases}\Delta \chi_{0,0}=\phi_{0,0} & \text { in } \Omega, \\ \frac{\partial \chi_{0,0}}{\partial \nu}=0 & \text { on } \partial \Omega, \\ \left(\chi_{0,0}\right)_{\Omega}=0, & \end{cases}
$$

respectively. For convenience we normalize the various fields by $\|J\|$ (we assume $\|J\|>0)$ :

$$
\tilde{\chi}_{0}=\frac{\chi_{0}}{\|J\|} \quad ; \quad \tilde{\phi}_{0}=\frac{\phi_{0}}{\|J\|}
$$

and

$$
\tilde{\chi}_{0,0}=\frac{\chi_{0,0}}{\|J\|} \quad ; \quad \tilde{\phi}_{0,0}=\frac{\phi_{0,0}}{\|J\|} .
$$

Then we set

$$
\tilde{\chi}_{0}=\tilde{\chi}_{0,0}+\omega_{\delta} \quad ; \quad \tilde{\phi}_{0}=\tilde{\phi}_{0,0}+\varphi_{\delta} .
$$

We begin by making the trivial observation that $\left(\tilde{\phi}_{0,0}, \tilde{\chi}_{0,0}\right) \in \mathcal{W}_{1}$, i.e.,

$$
\left\|\tilde{\chi}_{0,0}\right\|_{3,2}+\left\|\tilde{\phi}_{0,0}\right\|_{3,2} \leq C .
$$

We then define $F: \mathcal{W}_{1} \times \mathbb{R} \rightarrow H^{1}\left(\Omega, \mathbb{R}^{2}\right)$ by

$$
F\left(\varphi_{\delta}, \omega_{\delta}, \delta\right)=\left[\begin{array}{c}
-\sigma \Delta \varphi_{\delta}+\varphi_{\delta}-\delta^{2}\left|\nabla \tilde{\chi}_{0}\right|^{2} \tilde{\phi}_{0} \\
-\Delta \omega_{\delta}+\varphi_{\delta}-\delta^{2}\left|\nabla \tilde{\chi}_{0}\right|^{2} \tilde{\phi}_{0}+\delta^{2} \operatorname{div}\left(\left|\nabla \tilde{\chi}_{0}\right|^{2} \nabla \tilde{\chi}_{0}\right)
\end{array}\right] .
$$

Note that (14) provides a one-to-one correspondence between the solutions of $F\left(\varphi_{\delta}, \omega_{\delta}, \delta\right)=$ 0 and the solutions of (9). It can be easily verified that $F$ is well-defined because, by Sobolev embeddings,

$$
\begin{aligned}
& \left\|\left|\nabla \tilde{\chi}_{0}\right|^{2} \tilde{\phi}_{0}\right\|_{1,2}+\left\|\operatorname{div}\left(\left|\nabla \tilde{\chi}_{0}\right|^{2} \nabla \tilde{\chi}_{0}\right)\right\|_{1,2} \leq C\left(\left[\left\|\nabla \tilde{\chi}_{0}\right\|_{\infty}^{2}\left\|\tilde{\phi}_{0}\right\|_{1,2}\right.\right. \\
& \left.+\left\|\nabla \tilde{\chi}_{0}\right\|_{\infty}\left\|\tilde{\chi}_{0}\right\|_{2,2}\left\|\tilde{\phi}_{0}\right\|_{\infty}+\left\|\nabla \tilde{\chi}_{0}\right\|_{\infty}^{2}\left\|\tilde{\chi}_{0}\right\|_{3,2}+\left\|\nabla \tilde{\chi}_{0}\right\|_{\infty}\left\|\tilde{\chi}_{0}\right\|_{2,4}^{2}\right] \\
& \leq C\left[\left\|\tilde{\chi}_{0}\right\|_{3,2}^{3}+\left\|\tilde{\chi}_{0}\right\|_{3,2}^{2}\left\|\tilde{\phi}_{0}\right\|_{2,2}\right] .
\end{aligned}
$$

Furthermore, we have $F(0,0,0)=0$.

Let $\mathcal{D}: \mathcal{W}_{1} \times \mathbb{R} \rightarrow H^{1}\left(\Omega, \mathbb{R}^{2}\right)$ denote the linear operator

$$
\mathcal{D}(\varphi, \omega, \delta)=\left[\begin{array}{c}
\mathcal{D}_{1}(\varphi, \omega, \delta) \\
\mathcal{D}_{2}(\varphi, \omega, \delta)
\end{array}\right]
$$

where

$$
\mathcal{D}_{1}(\varphi, \omega, \delta)=-\sigma \Delta \varphi+\varphi-2 \delta^{2} \tilde{\phi}_{0} \nabla \tilde{\chi}_{0} \cdot \nabla \omega-\delta^{2}\left|\nabla \tilde{\chi}_{0}\right|^{2} \varphi
$$

and

$$
\begin{aligned}
\mathcal{D}_{2}(\varphi, \omega, \delta)=-\Delta \omega+\varphi- & 2 \delta^{2} \tilde{\phi}_{0} \nabla \tilde{\chi}_{0} \cdot \nabla \omega \\
& -\delta^{2}\left|\nabla \tilde{\chi}_{0}\right|^{2} \varphi+\delta^{2} \operatorname{div}\left(\left|\nabla \tilde{\chi}_{0}\right|^{2} \nabla \omega+2\left(\nabla \tilde{\chi}_{0} \cdot \nabla \omega\right) \nabla \tilde{\chi}_{0}\right)
\end{aligned}
$$


By using the same approach that we used to show that $F$ is well-defined, it can be verified that

$$
\begin{aligned}
\|\mathcal{D}(\varphi, \omega, \delta)\|_{1,2} \leq C\left[\delta^{2}\left\|\tilde{\chi}_{0}\right\|_{3,2}^{2}\|\omega\|_{3,2}+\|\varphi\|_{3,2}+\|\omega\|_{3,2}\right. \\
\left.+\delta^{2}\left\|\tilde{\phi}_{0}\right\|_{3,2}\|\omega\|_{3,2}\left\|\tilde{\chi}_{0}\right\|_{3,2}+\delta^{2}\left\|\tilde{\chi}_{0}\right\|_{3,2}^{2}\|\varphi\|_{3,2}\right] .
\end{aligned}
$$

Similarly, we can demonstrate that for any $\left(\tilde{\phi}_{0}, \tilde{\chi}_{0}\right) \in \mathcal{W}_{1}$ and $(\varphi, \omega) \in \mathcal{W}_{1}$ we have

$$
\begin{aligned}
& \left\|F\left(\varphi_{\delta}+\varphi, \omega_{\delta}+\omega, \delta\right)-F\left(\varphi_{\delta}, \omega_{\delta}, \delta\right)-\mathcal{D}(\varphi, \omega, \delta)\right\|_{1,2} \leq \\
& C \delta^{2}\left[\left(\left\|\tilde{\chi}_{0}\right\|_{3,2}+\left\|\tilde{\phi}_{0}\right\|_{3,2}\right)\|\omega\|_{3,2}^{2}+\left\|\tilde{\chi}_{0}\right\|_{3,2}\|\omega\|_{3,2}\|\varphi\|_{3,2}+\|\omega\|_{3,2}^{2}\left(\|\omega\|_{3,2}+\|\varphi\|_{3,2}\right)\right] .
\end{aligned}
$$

From the above inequality and (17) it follows that $\mathcal{D}$ is the Fréchet derivative of $F$ with respect to $\left(\varphi_{\delta}, \omega_{\delta}\right)$, and that it is continuous in any neighborhood of $(0,0,0)$ in $\mathcal{W}_{1} \times \mathbb{R}$.

We can now conclude from (16) that at $(0,0,0)$,

$$
D F(\varphi, \omega, 0)=\left[\begin{array}{c}
-\sigma \Delta \varphi+\varphi \\
-\Delta \omega+\varphi
\end{array}\right]
$$

It can be easily shown that $D F: \mathcal{W}_{1} \rightarrow H^{1}\left(\Omega, \mathbb{R}^{2}\right)$ is invertible. Since $(-\sigma \Delta+1)$ : $\mathcal{H}_{1} \rightarrow H^{1}(\Omega)$ and $-\Delta: \mathcal{H}_{2} \rightarrow H^{1}(\Omega)$ are both invertible, we have

$$
\left.\left\|(D F)^{-1}\right\| \leq\left\|(-\sigma \Delta+1)^{-1}\right\|+\|(-\Delta)^{-1}\right) \|\left(1+\left\|(-\sigma \Delta+1)^{-1}\right\|\right) .
$$

(Note that $-\Delta$ is invertible since the average of $\omega$ in $\Omega$ must vanish.) Consequently, by the implicit function theorem (cf. [16], for instance) we can find $\delta_{0}>0$ such that for every $0<\delta<\delta_{0}$ there exists $\left(\varphi_{\delta}, \omega_{\delta}\right) \in \mathcal{W}_{1}$ for which $F\left(\varphi_{\delta}, \omega_{\delta}, \delta\right)=0$. It readily follows that $\left(\varphi_{\delta}, \omega_{\delta}\right)$ converges in $H^{3}(\Omega) \times H^{3}(\Omega)$ to $(0,0)$ as $\delta \rightarrow 0$. In particular, we obtain that

$$
\left\|\omega_{\delta}\right\|_{3,2}+\left\|\varphi_{\delta}\right\|_{3,2} \underset{\delta \rightarrow 0}{\longrightarrow} 0 .
$$

Combining the above with (15) completes the proof of (10). The proof of (11) follows as well, since

$$
\left\|\left(1-\rho_{0}\right)\right\|_{2,2} \leq C \delta^{2}\left(\left\|\nabla \tilde{\chi}_{0}\right\|_{\infty}\left\|\tilde{\chi}_{0}\right\|_{3,2}+\left\|\tilde{\chi}_{0}\right\|_{2,4}^{2}\right) .
$$

Remark 2. Note that $\delta_{0}$ is independent of $\epsilon$ because $F\left(\varphi_{\delta}, \omega_{\delta}, \delta\right)$ is independent of $\epsilon$ as well.

Next, we show

Theorem 2. Let $\delta=\|J\| \epsilon$. There exists a $\delta_{0}>0$ such that (6) possesses a unique solution $(u, \phi)$ satisfying $\left\|u-u_{0}\right\|_{1,2}<\delta \epsilon$ for all $0<\epsilon \leq 1$ and $0<\delta<\delta_{0}$. Furthermore, there exists a constant $C=C(\Omega, \sigma)$, independent of both $\epsilon$ and $\delta$ such that

$$
\left\|\rho-\rho_{0}\right\|_{2}+\frac{1}{\|J\|}\left(\left\|\chi-\chi_{0}\right\|_{2}+\left\|\phi-\phi_{0}\right\|_{2}\right) \leq C \delta^{2} \epsilon^{2},
$$


and

$$
\left\|\rho-\rho_{0}\right\|_{\infty}+\left\|\rho-\rho_{0}\right\|_{1,2}+\epsilon\left\|\rho-\rho_{0}\right\|_{2,2}+\frac{1}{\|J\|}\left(\left\|\chi-\chi_{0}\right\|_{2,2}+\left\|\phi-\phi_{0}\right\|_{2,2}\right) \leq C \delta^{2} \epsilon .
$$

Here $u=\rho e^{i \chi}$.

Proof. We use Banach fixed point theorem in order to prove both existence and uniqueness. Recall the definition of $\tilde{\chi}_{0}$ and $\tilde{\phi}_{0}$ and set

$$
\rho_{1}=\rho-\rho_{0} \quad ; \quad \chi_{1}=\frac{1}{\|J\|}\left(\chi-\chi_{0}\right) \quad ; \quad \phi_{1}=\frac{1}{\|J\|}\left(\phi-\phi_{0}\right) .
$$

It is easy to show that (17) can be written in the equivalent form

$$
\begin{cases}\left(-\Delta+\|J\|^{2}\left|\nabla \tilde{\chi}_{0}\right|^{2}+\frac{1}{\epsilon^{2}}\left(3 \rho_{0}^{2}-1\right)\right) \rho_{1}+2\|J\|^{2} \rho_{0} \nabla \tilde{\chi}_{0} \cdot \nabla \chi_{1}=\Delta \rho_{0} & \\ -\|J\|^{2}\left|\nabla \chi_{1}\right|^{2} \rho_{0}-\|J\|^{2} \nabla \chi_{1} \cdot\left(2 \nabla \tilde{\chi}_{0} \nabla \chi_{1}\right) \rho_{1}-\frac{1}{\epsilon^{2}}\left(3 \rho_{0}+\rho_{1}\right) \rho_{1}^{2} & \text { in } \Omega \\ -\operatorname{div}\left(\rho_{0}^{2} \nabla \chi_{1}\right)-2 \operatorname{div}\left(\rho_{1} \rho_{0} \nabla \tilde{\chi}_{0}\right)+\rho_{0}^{2} \phi_{1}+2 \rho_{0} \rho_{1} \tilde{\phi}_{0}=-\rho_{1}\left(2 \rho_{0}+\rho_{1}\right) \phi_{1} & \\ -\rho_{1}^{2} \tilde{\phi}_{0}+\operatorname{div}\left(\rho_{1}^{2} \nabla \tilde{\chi}_{0}\right)+\operatorname{div}\left(\rho_{1}\left(2 \rho_{0}+\rho_{1}\right) \nabla \chi_{1}\right) & \text { in } \Omega \\ -\sigma \Delta \phi_{1}+\rho_{0}^{2} \phi_{1}+2 \rho_{0} \tilde{\phi}_{0} \rho_{1}=-\rho_{1}\left(2 \rho_{0}+\rho_{1}\right) \phi_{1}-\rho_{1}^{2} \tilde{\phi}_{0} & \text { in } \Omega \\ \frac{\partial \rho_{1}}{\partial \nu}=\frac{\partial \chi_{1}}{\partial \nu}=\frac{\partial \phi_{1}}{\partial \nu}=0 & \text { on } \partial \Omega\end{cases}
$$

We define the space

$$
\mathcal{H}=\left\{(\eta, \omega, \varphi) \in H^{2}\left(\Omega, \mathbb{R}^{3}\right)|(\nabla \eta, \nabla \omega, \nabla \varphi) \cdot \nu|_{\partial \Omega}=0 ;(\omega)_{\Omega}=0\right\},
$$

and let $(\eta, \omega, \varphi) \in \mathcal{H}$ be the weak solution of the following boundary value problem

$$
\begin{cases}-\left(\Delta-\|J\|^{2}\left|\nabla \tilde{\chi}_{0}\right|^{2}-\frac{1}{\epsilon^{2}}\left(3 \rho_{0}^{2}-1\right)\right) \eta+2\|J\|^{2} \rho_{0} \nabla \tilde{\chi}_{0} \cdot \nabla \omega=f_{1} & \text { in } \Omega \\ -\operatorname{div}\left(\rho_{0}^{2} \nabla \omega\right)-2 \operatorname{div}\left(\eta \rho_{0} \nabla \tilde{\chi}_{0}\right)+\rho_{0}^{2} \varphi+2 \rho_{0} \eta \tilde{\phi}_{0}=f_{2} & \text { in } \Omega \\ -\sigma \Delta \varphi+\rho_{0}^{2} \varphi+2 \rho_{0} \tilde{\phi}_{0} \eta=f_{3} & \text { in } \Omega,\end{cases}
$$

where $\left(f_{1}, f_{2}, f_{3}\right) \in L^{2}\left(\Omega, \mathbb{R}^{3}\right)$.

Step 1: Prove that $v=(\eta, \omega, \varphi)$ is well defined. To this end we use the LaxMilgram lemma. Let $w=(\tilde{\eta}, \tilde{\omega}, \tilde{\varphi})$. Define the bilinear form $B: \mathcal{H} \times \mathcal{H} \rightarrow \mathbb{R}$

$$
\begin{aligned}
B[v, w]= & \langle\nabla \eta, \nabla \tilde{\eta}\rangle+\left\langle\left(\|J\|^{2}\left|\nabla \tilde{\chi}_{0}\right|^{2}+\frac{1}{\epsilon^{2}}\left(3 \rho_{0}^{2}-1\right)\right) \eta, \tilde{\eta}\right\rangle \\
& +\|J\|^{2}\left[2\left\langle\rho_{0} \nabla \omega, \tilde{\eta} \nabla \tilde{\chi}_{0}\right\rangle+\left\langle\rho_{0} \nabla \omega, \rho_{0} \nabla \tilde{\omega}\right\rangle+2\left\langle\rho_{0} \nabla \tilde{\omega}, \eta \nabla \tilde{\chi}_{0}\right\rangle\right. \\
& \left.+\left\langle\rho_{0} \tilde{\omega},\left(\rho_{0} \varphi+2 \eta \tilde{\phi}_{0}\right)\right\rangle+C_{0}\left(\langle\sigma \nabla \varphi, \nabla \tilde{\varphi}\rangle+\left\langle\rho_{0} \tilde{\varphi},\left(\rho_{0} \varphi+2 \eta \tilde{\phi}_{0}\right)\right\rangle\right)\right],
\end{aligned}
$$


where the (positive) value of $C_{0}$ will be determined later. Since by (10) both $\tilde{\chi}_{0}$ and $\tilde{\phi}_{0}$ are in $H^{3}(\Omega)$, it readily follows from Sobolev embeddings that there exists $C(\Omega, \epsilon)$ such that

$$
|B[v, w]| \leq C\|v\|_{1,2}\|w\|_{1,2} .
$$

To use the Lax-Milgram Lemma we need yet to consider the quadratic form $B[v, v]$. Note that

$$
B[v, v]=I_{1}+I_{2}+I_{3}
$$

where

$$
\begin{aligned}
& I_{1}=\|\nabla \eta\|_{2}^{2}+\frac{1}{\epsilon^{2}}\left\|\left(3 \rho_{0}^{2}-1\right)^{1 / 2} \eta\right\|_{2}^{2}, \\
& I_{2}=\|J\|^{2}\left\{\left\|\rho_{0} \nabla \omega\right\|_{2}^{2}+4\left\langle\rho_{0} \eta \nabla \omega, \nabla \tilde{\chi}_{0}\right\rangle+\left\langle\rho_{0} \omega,\left(\rho_{0} \varphi+2 \eta \tilde{\phi}_{0}\right)\right\rangle\right\}, \\
& I_{3}=\|J\|^{2} C_{0}\left\{\sigma\|\nabla \varphi\|_{2}^{2}+\left\langle\rho_{0} \varphi,\left(\rho_{0} \varphi+2 \eta \tilde{\phi}_{0}\right)\right\rangle\right\} .
\end{aligned}
$$

With the aid of the Cauchy-Schwarz inequality we obtain for any $\delta_{1}>0$,

$$
\begin{aligned}
I_{2} \geq\|J\|^{2}\left\{\frac{1}{2}\left\|\rho_{0} \nabla \omega\right\|_{2}^{2}-8\left\|\nabla \tilde{\chi}_{0}\right\|_{\infty}^{2}\|\eta\|_{2}^{2}-\right. & 2 \delta_{1}\left\|\rho_{0}\right\|_{\infty}^{2}\|\omega\|_{2}^{2} \\
& \left.-\left(\frac{\left\|\rho_{0}\right\|_{\infty}^{2}}{4 \delta_{1}}\right)\|\varphi\|_{2}^{2}-\frac{\left\|\tilde{\phi}_{0}\right\|_{\infty}^{2}}{\delta_{1}}\|\eta\|_{2}^{2}\right\} .
\end{aligned}
$$

Applying Cauchy-Schwarz inequality to $I_{3}$ yields,

$$
I_{3} \geq\|J\|^{2} C_{0}\left\{\sigma\|\nabla \varphi\|_{2}^{2}+\frac{1}{2}\left\|\rho_{0} \varphi\right\|_{2}^{2}-2\left\|\tilde{\phi}_{0}\right\|_{\infty}^{2}\|\eta\|_{2}^{2}\right\} .
$$

By (11) and Sobolev embeddings we have that

$$
\left\|1-\rho_{0}\right\|_{\infty} \leq C \delta^{2} \Longrightarrow\left\|\left(3 \rho_{0}^{2}-1\right)-2\right\|_{\infty} \leq C \delta^{2} .
$$

Furthermore, by the Poincare's inequality (recall that $(\omega)_{\Omega}=0$ ), there exists a $\lambda=\lambda(\Omega)>0$ such that

$$
\|\nabla \omega\|_{2}^{2} \geq \lambda\|\omega\|_{2}^{2} .
$$

Using (24)-(26), (27) and setting $\delta_{1}=\lambda / 8$ and $C_{0}=2 / \delta_{1}$, we deduce the existence of $C(\Omega)$ such that, for a sufficiently small $\delta$ the inequality

$$
|B[v, v]| \geq C \frac{\delta^{2}}{\epsilon^{2}}\left(\|\omega\|_{1,2}^{2}+\|\varphi\|_{1,2}^{2}\right)+\frac{1}{\epsilon^{2}}\|\eta\|_{2}^{2}+\|\nabla \eta\|_{2}^{2}
$$

holds. We can thus conclude the existence of a unique $v \in \mathcal{H}$ such that

$$
B[v, w]=\langle F, w\rangle \quad \forall w \in \mathcal{H},
$$

where $F=\left(f_{1},\|J\|^{2} f_{2}, C_{0}\|J\|^{2} f_{3}\right)$. 
Let $\left(\rho-\rho_{0}, \chi-\tilde{\chi}_{0}, \phi-\tilde{\phi}_{0}\right) \in \mathcal{H}$. We set

$$
\begin{aligned}
& f_{1}=\Delta \rho_{0}-\|J\|^{2}\left|\nabla \chi_{1}\right|^{2} \rho_{0}-\left\|J^{2}\right\| \nabla \chi_{1} \cdot\left(2 \nabla \tilde{\chi}_{0}+\nabla \chi_{1}\right) \rho_{1}-\frac{1}{\epsilon^{2}}\left(3 \rho_{0}+\rho_{1}\right) \rho_{1}^{2} \\
& f_{2}=-\rho_{1}\left(2 \rho_{0}+\rho_{1}\right) \phi_{1}-\rho_{1}^{2} \tilde{\phi}_{0}+\operatorname{div}\left(\rho_{1}^{2} \nabla \tilde{\chi}_{0}\right)+\operatorname{div}\left(\rho_{1}\left(2 \rho_{0}+\rho_{1}\right) \nabla \chi_{1}\right) \\
& f_{3}=-\rho_{1}\left(2 \rho_{0}+\rho_{1}\right) \phi_{1}-\rho_{1}^{2} \tilde{\phi}_{0} .
\end{aligned}
$$

Substituting the above into (22), we can define the operator $\mathcal{A}: \mathcal{H} \rightarrow \mathcal{H}$

$$
\mathcal{A}\left(\rho_{1}, \chi_{1}, \phi_{1}\right)=(\eta, \omega, \varphi) .
$$

We look for a fixed point of $\mathcal{A}$. We equip $\mathcal{H}$ with the norm

$$
\|w\|_{\mathcal{H}}=\|\eta\|_{1,2}+\|\eta\|_{\infty}+\epsilon\left\|D^{2} \eta\right\|_{2}+\|\omega\|_{2,2}+\|\varphi\|_{2,2} .
$$

Step 2: Let $v=\left(\rho_{1}, \chi_{1}, \phi_{1}\right)$. We prove that for sufficiently small $\epsilon$ and $\delta$ there exist $C(\Omega, \sigma)$ and $r(\epsilon, \delta) \leq C \delta \epsilon$ for which

$$
v \in B(0, r) \Rightarrow A(v) \in B(0, r) .
$$

Let then $0<r \leq \epsilon$ and $v \in B(0, r)$. We begin by deriving a bound on $\|\eta\|_{2}$ and $\|A(v)\|_{1,2}$. By (29), (10), and (27) we have that

$\left\|f_{1}\right\|_{2}^{2} \leq C\left\{\left\|\Delta \rho_{0}\right\|_{2}^{2}+\|J\|^{4}\left[\left\|\nabla \chi_{1}\right\|_{4}^{4}+\left\|\rho_{1}\right\|_{4}^{2}\left(\left\|\nabla \chi_{1}\right\|_{4}^{2}+\left\|\nabla \chi_{1}\right\|_{8}^{4}\right)\right]+\frac{1}{\epsilon^{4}}\left(\left\|\rho_{1}\right\|_{4}^{4}+\left\|\rho_{1}\right\|_{6}^{6}\right)\right\}$.

Recall that, by (11), there exists $C(\Omega, \sigma)>0$, such that for a sufficiently small $\delta>0$ we have

$$
\left\|\Delta \rho_{0}\right\|_{2} \leq C \delta^{2}
$$

Sobolev embeddings then yield

$$
\left\|f_{1}\right\|_{2} \leq C\left(\delta^{2}+\frac{r^{2}}{\epsilon^{2}}\right) .
$$

Similarly, we obtain that

$$
\begin{aligned}
\left|\left\langle\omega, f_{2}\right\rangle+\left\langle\varphi, f_{3}\right\rangle\right| \leq C\left\{\left[\left(\left\|\rho_{1}\right\|_{4}+\left\|\rho_{1}\right\|_{8}^{2}\right)\left\|\phi_{1}\right\|_{4}+\left\|\rho_{1}\right\|_{4}^{2}\right]\left(\|\omega\|_{2}+\|\varphi\|_{2}\right)\right. \\
\left.+\left[\left\|\rho_{1}\right\|_{4}^{2}+\left(\left\|\rho_{1}\right\|_{4}+\left\|\rho_{1}\right\|_{8}^{2}\right)\left\|\nabla \chi_{1}\right\|_{4}\right]\|\nabla \omega\|_{2}\right\}
\end{aligned}
$$

and hence,

$$
\left|\left\langle\omega, f_{2}\right\rangle+\left\langle\varphi, f_{3}\right\rangle\right| \leq C r^{2}\left(\|\omega\|_{1,2}+\|\varphi\|_{2}\right) .
$$

Combining the above with (31) yields

$$
|\langle\mathcal{A}(v), F\rangle| \leq C\left[\left(\delta^{2}+\frac{r^{2}}{\epsilon^{2}}\right)\|\eta\|_{2}+\frac{\delta^{2} r^{2}}{\epsilon^{2}}\left(\|\omega\|_{1,2}+\|\varphi\|_{2}\right)\right] .
$$


As $B(\mathcal{A}(v), \mathcal{A}(v))=\langle\mathcal{A}(v), F\rangle$ we obtain by (28) that

$$
\|\eta\|_{2} \leq C\left[\delta^{2} \epsilon^{2}+r^{2}\right] .
$$

Upon multiplying (22) by $\omega$ and (22k) by $\varphi$ we sum the resulting equations and integrate over $\Omega$ to obtain

$$
\|\nabla \omega\|_{2}^{2}+\sigma\|\nabla \varphi\|_{2}^{2}+\|\varphi\|_{2}^{2} \leq C\left[r^{2}\left(\|\omega\|_{1,2}+\|\varphi\|_{2}\right)+\|\eta\|_{2}\left(\|\omega\|_{1,2}+\|\varphi\|_{2}\right)\right] .
$$

Using Poincare's inequality we then obtain, with the aid of (33), that

$$
\|\omega\|_{1,2}+\|\varphi\|_{1,2} \leq C\left(r^{2}+\delta^{2} \epsilon^{2}\right) .
$$

Substituting the above, together with (33) into (32) and using (28) yields

$$
\|\nabla \eta\|_{2} \leq \frac{C}{\epsilon}\left(r^{2}+\delta^{2} \epsilon^{2}\right) .
$$

To complete the proof of (30) we rewrite first (22b) in the form

$$
-\operatorname{div}\left(\rho_{0}^{2} \nabla \omega\right)=2 \operatorname{div}\left(\eta \rho_{0} \nabla \tilde{\chi}_{0}\right)-\rho_{0}^{2} \varphi-2 \rho_{0} \eta \tilde{\phi}_{0}+f_{2} .
$$

We attempt to estimate the $L_{2}$-norm of the right-hand side. Clearly

$$
\left\|2 \operatorname{div}\left(\eta \rho_{0} \nabla \tilde{\chi}_{0}\right)-\rho_{0}^{2} \varphi-2 \rho_{0} \eta \tilde{\phi}_{0}\right\|_{2} \leq C\left(\|\eta\|_{1,2}+\|\varphi\|_{2}\right) \leq \frac{C}{\epsilon}\left(r^{2}+\delta^{2} \epsilon^{2}\right) .
$$

Furthermore, we have

$$
\left\|\rho_{1}\left(2 \rho_{0}+\rho_{1}\right) \phi_{1}+\rho_{1}^{2} \tilde{\phi}_{0}\right\|_{2} \leq C\left[\left(\left\|\rho_{1}\right\|_{4}+\left\|\rho_{1}\right\|_{8}^{2}\right)\left\|\phi_{1}\right\|_{4}+\left\|\rho_{1}\right\|_{4}^{2}\right] \leq C r^{2}
$$

and

$$
\begin{aligned}
& \left\|\operatorname{div}\left(\rho_{1}^{2} \nabla \tilde{\chi}_{0}\right)\right\|_{2}+\left\|\operatorname{div}\left(\rho_{1}\left(2 \rho_{0}+\rho_{1}\right) \nabla \chi_{1}\right)\right\|_{2} \leq \\
& \quad C\left(\left\|\rho_{1}\right\|_{4}^{2}+\left\|\nabla \rho_{1}\right\|_{2}\left\|\rho_{1}\right\|_{\infty}+\left\|\rho_{1}\right\|_{\infty}\left\|\chi_{1}\right\|_{2,2}+\left\|\nabla \rho_{1}\right\|_{2}\left\|\nabla \chi_{1}\right\|_{2}\right) \leq C r^{2} .
\end{aligned}
$$

Combining the above with (37) and (36) yields for $\epsilon \leq 1$,

$$
\left\|\operatorname{div}\left(\rho_{0}^{2} \nabla \omega\right)\right\|_{2} \leq \frac{C}{\epsilon}\left(r^{2}+\delta^{2} \epsilon^{2}\right) .
$$

Writing $\operatorname{div}\left(\rho_{0}^{2} \nabla \omega\right)=\rho_{0}^{2} \Delta \omega+2 \rho_{0} \nabla \rho_{0} \cdot \nabla \omega$, using (11) and standard elliptic estimates, we obtain from (38) that

$$
\|\omega\|_{2,2} \leq \frac{C}{\epsilon}\left(r^{2}+\delta^{2} \epsilon^{2}\right) .
$$

In a similar manner it is possible to show that

$$
\|\varphi\|_{2,2} \leq C\left(r^{2}+\delta^{2} \epsilon^{2}\right)
$$


To complete the proof we need yet to bound $\epsilon\left\|D^{2} \eta\right\|_{2}$ and $\|\eta\|_{\infty}$. To this end we rewrite $(22 \mathrm{a})$ in the form

$$
-\Delta \eta=-\left(\|J\|^{2}\left|\nabla \tilde{\chi}_{0}\right|^{2}-\frac{1}{\epsilon^{2}}\left(3 \rho_{0}^{2}-1\right)\right) \eta-2\|J\|^{2} \rho_{0} \nabla \tilde{\chi}_{0} \cdot \nabla \omega+f_{1} .
$$

It easily follows that for sufficiently small $\delta$ we have

$$
\left\|\left(\|J\|^{2}\left|\nabla \tilde{\chi}_{0}\right|^{2}-\frac{1}{\epsilon^{2}}\left(3 \rho_{0}^{2}-1\right)\right) \eta\right\|_{2} \leq \frac{C}{\epsilon^{2}}\left(r^{2}+\delta^{2} \epsilon^{2}\right) .
$$

Furthermore, as

$$
\|J\|^{2}\left\|\rho_{0} \nabla \tilde{\chi}_{0} \cdot \nabla \omega\right\|_{2} \leq C \frac{\delta^{2}}{\epsilon^{2}} r^{2},
$$

we obtain with the aid of (41) and (31) that

$$
\|\eta\|_{2,2} \leq \frac{C}{\epsilon^{2}}\left(r^{2}+\delta^{2} \epsilon^{2}\right) .
$$

It follows from Agmon's inequality (cf. [17, Lemma 13.2]) in conjunction with (33) that

$$
\|\eta\|_{\infty} \leq C\|\eta\|_{2}^{1 / 2}\|\eta\|_{2,2}^{1 / 2} \leq \frac{C}{\epsilon}\left(r^{2}+\delta^{2} \epsilon^{2}\right)
$$

Combining the above with (33), (35), (39), (42), and (40) yields

$$
\|\mathcal{A}(v)\|_{\mathcal{H}} \leq \frac{C}{\epsilon}\left(r^{2}+\delta^{2} \epsilon^{2}\right) .
$$

We may thus choose $r=\delta \epsilon$ to obtain, for a sufficiently small value of $\delta$, that

$$
\|\mathcal{A}(v)\|_{\mathcal{H}} \leq C \epsilon \delta^{2}<r .
$$

Step 3: Let $\left(v_{1}, v_{2}\right) \in B(0, r)^{2}$. We prove that there exists $\gamma<1$ such that

$$
\left\|\mathcal{A}\left(v_{1}\right)-\mathcal{A}\left(v_{2}\right)\right\|_{\mathcal{H}} \leq \gamma\left\|v_{1}-v_{2}\right\|_{\mathcal{H}} .
$$

It can be easily verified that

$$
\begin{aligned}
& \left\|f_{1}\left(v_{1}\right)-f_{1}\left(v_{2}\right)\right\|_{2} \leq C\left(\|J\|^{2}+\frac{1}{\epsilon^{2}}\right) r\left\|v_{1}-v_{2}\right\|_{\mathcal{H}}, \\
& \left\|f_{2}\left(v_{1}\right)-f_{2}\left(v_{2}\right)\right\|_{2} \leq C r\left\|v_{1}-v_{2}\right\|_{\mathcal{H}}, \\
& \left\|f_{2}\left(v_{1}\right)-f_{2}\left(v_{2}\right)\right\|_{2} \leq C r\left\|v_{1}-v_{2}\right\|_{\mathcal{H}} .
\end{aligned}
$$

Let now $\mathcal{A}\left(v_{1}\right)=\left(\eta_{1}, \omega_{1}, \varphi_{1}\right)$ and $\mathcal{A}\left(v_{2}\right)=\left(\eta_{2}, \omega_{2}, \varphi_{2}\right)$. As

$$
B\left(\mathcal{A}\left(v_{1}\right)-\mathcal{A}\left(v_{2}\right), \mathcal{A}\left(v_{1}\right)-\mathcal{A}\left(v_{2}\right)\right)=\left\langle\mathcal{A}\left(v_{1}\right)-\mathcal{A}\left(v_{2}\right), F\left(v_{1}\right)-F\left(v_{2}\right)\right\rangle,
$$

we obtain by (28) that

$$
\left\|\eta_{1}-\eta_{2}\right\|_{2} \leq C r\left\|v_{1}-v_{2}\right\|_{\mathcal{H}}
$$


The same procedure that led to (34) and (35) enables us to conclude that

$$
\left\|\omega_{1}-\omega_{2}\right\|_{1,2}+\left\|\varphi_{1}-\varphi_{2}\right\|_{1,2} \leq C r\left\|v_{1}-v_{2}\right\|_{\mathcal{H}},
$$

and that

$$
\left\|\nabla\left(\eta_{1}-\eta_{2}\right)\right\|_{2} \leq C \frac{r}{\epsilon}\left\|v_{1}-v_{2}\right\|_{\mathcal{H}} .
$$

We then proceed in precisely the same manner as in the derivation of (39) and (40) to obtain that

$$
\epsilon\left\|\omega_{1}-\omega_{2}\right\|_{2,2}+\left\|\varphi_{1}-\varphi_{2}\right\|_{2,2} \leq C r\left\|v_{1}-v_{2}\right\|_{\mathcal{H}} .
$$

Finally, using the same procedure as in the derivation of (43) and (42) we obtain that

$$
\left\|\eta_{1}-\eta_{2}\right\|_{\infty}+\epsilon^{2}\left\|\eta_{1}-\eta_{2}\right\|_{2,2} \leq C \frac{r}{\epsilon}\left\|v_{1}-v_{2}\right\|_{\mathcal{H}}
$$

Combining all of the above then yields

$$
\left\|\mathcal{A}\left(v_{1}\right)-\mathcal{A}\left(v_{2}\right)\right\|_{\mathcal{H}} \leq C \frac{r}{\epsilon}\left\|v_{1}-v_{2}\right\|_{\mathcal{H}},
$$

and since $r=\delta \epsilon$, we obtain (44) for a sufficiently small value of $\delta$.

\section{$3 \quad$ Linear stability}

In what follows, we examine the linear stability of the solution we have obtained in the previous section. To this end, let

$$
\mathcal{U}=\left\{u \in H^{2}(\Omega, \mathbb{C}): \partial u /\left.\partial \nu\right|_{\partial \Omega}=0\right\}
$$

and define the non-linear operator $\mathcal{L}_{\epsilon}: \mathcal{U} \rightarrow L^{2}(\Omega, \mathbb{C})$ by

$$
\mathcal{L}_{\epsilon} u=-\Delta u+i \phi u-\frac{u}{\epsilon^{2}}\left(1-|u|^{2}\right) .
$$

for any $u \in \mathcal{U}$. In the above $\phi$ denotes a non-local, non-linear operator of $u$. We define $\phi$, in view of (5), as the solution of

$$
\begin{cases}\sigma \Delta \phi=\operatorname{div}(\Im\{\bar{u} \nabla u\}) & \text { in } \Omega \\ \frac{\partial \phi}{\partial \nu}=-\frac{J}{\sigma} & \text { on } \partial \Omega \\ \left(|u|^{2} \phi\right)_{\Omega}=0 . & \end{cases}
$$

The system (3) can then be written in the form

$$
u_{t}+\mathcal{L}_{\epsilon} u=0
$$

We look for the spectrum of $\mathcal{A}=D \mathcal{L}_{\epsilon}\left(u_{s}\right)$-the Fréchet derivative of $\mathcal{L}_{\epsilon}$ at $u_{s}$. Set $\phi_{s}=\phi\left(u_{s}\right)$. It can be readily verified that

$$
\mathcal{A} u=-\Delta u+i\left(\phi_{s} u+\hat{\varphi} u_{s}\right)-\frac{u}{\epsilon^{2}}\left(1-\rho_{s}^{2}\right)+\frac{2 u_{s}}{\epsilon^{2}} \Re\left(\bar{u}_{s} u\right),
$$


where $\hat{\varphi}\left(u, u_{s}\right)$ is a non-local linear operator given by the solution of

$$
\begin{cases}\sigma \Delta \hat{\varphi}=\operatorname{div}\left(\Im\left\{\bar{u}_{s} \nabla u+\bar{u} \nabla u_{s}\right\}\right) & \text { in } \Omega \\ \frac{\partial \hat{\varphi}}{\partial \nu}=0 & \text { on } \partial \Omega \\ \left.\left(\left|u_{s}\right|^{2} \hat{\varphi}+2 \phi_{s} \Re\left(\bar{u}_{s} u\right)\right]\right)_{\Omega}=0 . & \end{cases}
$$

Note that $\mathcal{A}$ has a non-trivial kernel, i.e., $\mathcal{A} i u_{s}=0$. This non-trivial kernel reflects the fact that

$$
e^{-i \Theta} \mathcal{L}_{\epsilon} e^{i \Theta}=\mathcal{L}_{\epsilon} .
$$

Let $\left\{u_{n}\right\}_{n=0}^{\infty}$ denote the system of eigenfunctions associated with $\mathcal{A}$ where $u_{0}=i u_{s}$. By Theorem 16.5 in [17] we have $\operatorname{span}\left\{u_{n}\right\}_{n=0}^{\infty}=L^{2}(\Omega, \mathbb{C})$ and we can thus set

$$
D(\mathcal{A})=\mathcal{U} \cap \overline{\operatorname{span}\left\{u_{n}\right\}_{n=1}^{\infty}}
$$

as the domain of $\mathcal{A}$, thereby eliminating $i u_{s}$ from the domain.

Let

$$
\tilde{u}=\tilde{\rho} e^{i \tilde{\chi}}=\left(\rho_{s}+\delta^{\prime} \rho\right) e^{i\left(\chi_{s}+\delta^{\prime} \chi\right)} \in \mathcal{U}
$$

denote an infinitesimal perturbation of $u_{s}$, where $\delta^{\prime}$ is a small parameter. Then, $\tilde{u}=u_{s}+\delta^{\prime} u+o\left(\delta^{\prime}\right)$ where

$$
u=e^{i \chi_{s}}\left(\rho+i \rho_{s} \chi\right) .
$$

Consider then the linear operator

$$
\mathcal{B}=e^{-i \chi_{s}} \mathcal{A} e^{i \chi_{s}},
$$

defined on $D(\mathcal{B})=e^{-i \chi_{s}} D(\mathcal{A})$. More explicitly, we have

$$
D(\mathcal{B})=\{v \in \mathcal{U} \mid
$$

Since $e^{i \chi_{s}}$ is a unitary operator, we have $\sigma(\mathcal{A})=\sigma(\mathcal{B})$. We write any $v \in D(\mathcal{B})$ as $v=e^{-i \chi_{s}} u$ with $u \in D(\mathcal{A})$. Substituting into (46) yields

$$
\begin{aligned}
\mathcal{B} v=-\Delta v+\left|\nabla \chi_{s}\right|^{2} v-\frac{1}{\epsilon^{2}}(1- & \left.\rho_{s}^{2}\right) v+\frac{2}{\epsilon^{2}} \rho_{s}^{2} \Re v \\
& +i\left(\left(-\Delta \chi_{s}\right) v-2 \nabla \chi_{s} \cdot \nabla v+\phi_{s} v+\rho_{s} \varphi(v)\right),
\end{aligned}
$$

where $\varphi=\varphi(v)$ is given (according to (47)) by the solution of

$$
\begin{cases}\sigma \Delta \varphi=\operatorname{div}\left(\Im\left(\rho_{s} \nabla v+\bar{v} \nabla \rho_{s}\right)+2 \rho_{s} \nabla \chi_{s} \Re v\right) & \text { in } \Omega \\ \frac{\partial \varphi}{\partial \nu}=0 & \text { on } \partial \Omega \\ \left(\rho_{s}^{2} \varphi+2 \phi_{s} \rho_{s} \Re v\right)_{\Omega}=0 . & \end{cases}
$$


In view of (48) we have $v=\rho+i \rho_{s} \chi$. Next we look for a non-trivial solution to the eigenvalue problem $\mathcal{B} v=\lambda v$, i.e.,

$$
\begin{aligned}
& \Re(\mathcal{B} v)=\Re(\lambda v) \\
& \Im(\mathcal{B} v)=\Im(\lambda v) .
\end{aligned}
$$

We now prove the stability of the solution of (6) in the neighborhood of $\left(\rho_{0} e^{i \chi_{0}}, \phi_{0}\right)$ where $\left(\rho_{0}, \chi_{0}, \phi_{0}\right)$ are given by (9). We establish this for a sufficiently small value of $\|J\| \epsilon$, which is precisely the limit where existence has been obtained in the previous section.

Proposition 2. Let $u_{s}$ denote a solution of (3) in the neighborhood of $\left(\rho_{0} e^{i \chi_{0}}, \phi_{0}\right)$ given by (9). Furthermore, let $\mathcal{A}=D \mathcal{L}_{\epsilon}\left(u_{s}\right): D(\mathcal{A}) \rightarrow L^{2}(\Omega, \mathbb{C})$. There exists $\delta_{0}>0$, such that for all $0<\epsilon \leq 1$ and $0<\delta<\delta_{0}$ we have

$$
\min _{\lambda \in \sigma(\mathcal{A})} \Re \lambda>0
$$

where $\delta=\|J\| \epsilon$.

Proof. Let $v=\rho+i \rho_{s} \chi \in D(\mathcal{B})$ denote an eigenfunction of $\mathcal{B}$ associated with the eigenvalue $\lambda$. By (49) and (51), the triplet $(\rho, \chi, \lambda)$ must satisfy the following problem

$$
\begin{cases}-\Delta \rho+\rho\left|\nabla \chi_{s}\right|^{2}+2 \rho_{s} \nabla \chi \cdot \nabla \chi_{s}-\frac{\rho}{\epsilon^{2}}\left(1-3 \rho_{s}^{2}\right)=\lambda_{r} \rho-\lambda_{i} \rho_{s} \chi & \text { in } \Omega \\ -\operatorname{div}\left(\rho_{s}^{2} \nabla \chi\right)-2 \operatorname{div}\left(\rho_{s} \rho \nabla \chi_{s}\right)+\rho_{s}^{2} \varphi+2 \rho_{s} \rho \phi_{s}=\lambda_{r} \rho_{s}^{2} \chi+\lambda_{i} \rho_{s} \rho & \text { in } \Omega \\ -\sigma \Delta \varphi+\operatorname{div}\left(\rho_{s}^{2} \nabla \chi\right)+2 \operatorname{div}\left(\rho_{s} \rho \nabla \chi_{s}\right)=0 & \text { in } \Omega \\ \frac{\partial \rho}{\partial \nu}=\frac{\partial \chi}{\partial \nu}=\frac{\partial \varphi}{\partial \nu}=0 & \text { on } \partial \Omega \\ \left(\rho_{s}^{2} \varphi+2 \phi_{s} \rho_{s} \rho\right)_{\Omega}=0, & \end{cases}
$$

where $\lambda_{r}=\Re \lambda$ and $\lambda_{i}=\Im \lambda$. Note that to obtain (53 a) we need to use (77b). Since the spectrum of $\mathcal{B}$ is discrete (cf. [17] chapter 15), it suffices to show that all critical values of $\lambda$, for which non-trivial solutions for the above problem exist (excluding, of course, $u=i u_{s}$ ), lie in the right hand side of $\mathbb{C}$.

Taking the inner product in $L^{2}(\Omega)$ of (53k) with $\sigma \varphi-\chi$ we obtain

$$
\|\nabla(\sigma \varphi-\chi)\|_{2}^{2}=\left\langle\left(\rho_{s}^{2}-1\right) \nabla \chi, \nabla(\sigma \varphi-\chi)\right\rangle+2\left\langle\rho_{s} \nabla(\sigma \varphi-\chi), \rho \nabla \chi_{s}\right\rangle .
$$

It can be easily demonstrated using (10), (20), and Sobolev embeddings that

$$
\left\|\rho \nabla \chi_{s}\right\|_{2} \leq\left\|\nabla \chi_{0}\right\|_{\infty}\|\rho\|_{2}+\left\|\nabla\left(\chi_{s}-\chi_{0}\right)\right\|_{p}\|\rho\|_{q} \leq C\|J\|\left(\|\rho\|_{2}+\delta^{2} \epsilon\|\rho\|_{1,2}\right) .
$$

In the above $p>2$ and $q=2 p /(p-2)$. Consequently, using (111) and Sobolev embeddings and recalling that $\|J\|=\delta / \epsilon$, it readily follows that

$$
\|\nabla(\sigma \varphi-\chi)\|_{2} \leq C\left(\delta^{2}\|\nabla \chi\|_{2}+\delta\left(\|\rho\|_{2} / \epsilon+\delta^{2}\|\rho\|_{1,2}\right)\right) .
$$


Next, we multiply (53) by $\chi$ and $(53 \mathrm{a})$ by $\rho$, then integrate their sum by parts to obtain

$$
\begin{aligned}
\left\|\rho_{s} \nabla \chi\right\|_{2}^{2}+ & \|\nabla \rho\|_{2}^{2}+4\left\langle\rho_{s} \nabla \chi, \rho \nabla \chi_{s}\right\rangle+\left\|\rho \nabla \chi_{s}\right\|_{2}^{2}+\frac{2}{\epsilon^{2}}\|\rho\|_{2}^{2} \\
& +\left\langle\rho_{s} \chi, \rho_{s} \varphi+2 \rho \phi_{s}\right\rangle \leq \frac{3}{\epsilon^{2}}\left\|1-\rho_{s}^{2}\right\|_{\infty}\|\rho\|_{2}^{2}+\lambda_{r}\left(\left\|\rho_{s} \chi\right\|_{2}^{2}+\|\rho\|_{2}^{2}\right) .
\end{aligned}
$$

We now write,

$$
\begin{aligned}
& \int_{\Omega} \rho_{s} \chi\left(\rho_{s} \varphi+2 \rho \phi_{s}\right)=\int_{\Omega} \rho_{s}(\chi-\sigma \varphi)\left(\rho_{s} \varphi+2 \rho \phi_{s}\right)+\int_{\Omega} \sigma \rho_{s} \varphi\left(\rho_{s} \varphi+2 \rho \phi_{s}\right) \\
& =\int_{\Omega} \rho_{s}(\chi-\sigma \varphi)\left(\rho_{s} \varphi+2 \rho \phi_{s}\right)+\sigma\left\|\rho_{s} \varphi+2 \rho \phi_{s}\right\|_{2}^{2}-2 \sigma \int_{\Omega} \rho \phi_{s}\left(\rho_{s} \varphi+2 \rho \phi_{s}\right),
\end{aligned}
$$

which together with (53) yields

$$
\begin{aligned}
\int_{\Omega} \rho_{s} \chi\left(\rho_{s} \varphi+2 \rho \phi_{s}\right) & \geq \sigma\left\|\rho_{s} \varphi+2 \rho \phi_{s}\right\|_{2}^{2} \\
- & \left(\left\|\rho_{s}\left[\sigma \phi-\chi-(\sigma \varphi-\chi)_{\Omega}\right]\right\|_{2}+2 \sigma\left\|\rho \phi_{s}\right\|_{2}\right)\left\|\rho_{s} \varphi+2 \rho \phi_{s}\right\|_{2}
\end{aligned}
$$

Applying Cauchy's inequality, $2 a b \leq 2 \alpha a^{2}+\frac{2}{\alpha} b^{2}$, with $\alpha=\frac{1}{4}$, leads to

$$
4\left\langle\rho_{s} \nabla \chi, \rho \nabla \chi_{s}\right\rangle \geq-\frac{1}{2}\left\|\rho_{s} \nabla \chi\right\|_{2}^{2}-8\left\|\rho \nabla \chi_{s}\right\|_{2}^{2} .
$$

Recall that by (10) and (19) we have that $\left\|\nabla \chi_{s}\right\|_{2} \leq C \delta / \epsilon$, and hence we obtain from (58) that

$$
4\left\langle\rho_{s} \nabla \chi, \rho \nabla \chi_{s}\right\rangle \geq-\frac{1}{2}\left\|\rho_{s} \nabla \chi\right\|_{2}^{2}-\frac{C \delta^{2}}{\epsilon^{2}} .
$$

Furthermore, because $\left\|1-\rho_{s}\right\|_{\infty} \leq C \delta^{2}$ by (11) and (20), we substitute (57) and (59) into (56) to obtain

$$
\begin{aligned}
\|\nabla \rho\|_{2}^{2}+\frac{1}{2}\|\nabla \chi\|_{2}^{2}+\sigma \| & \rho_{s} \varphi \\
+ & 2 \rho \phi_{s}\left\|_{2}^{2}+\frac{2}{\epsilon^{2}}\right\| \rho \|_{2}^{2} \\
\leq & C \delta^{2}\left[\frac{1}{\epsilon^{2}}\|\rho\|_{2}^{2}+\|\nabla \rho\|_{2}^{2}+\|\nabla \chi\|_{2}^{2}\right] \\
& +\left(\left\|\rho_{s}\left[\sigma \phi-\chi-(\sigma \varphi-\chi)_{\Omega}\right]\right\|_{2}\right. \\
& \left.+2 \sigma\left\|\phi_{s} \rho\right\|_{2}\right)\left\|\rho_{s} \varphi+2 \rho \phi_{s}\right\|_{2}+\lambda_{r}\left(\left\|\rho_{s} \chi\right\|_{2}^{2}+\|\rho\|_{2}^{2}\right) .
\end{aligned}
$$

By (10) and (19) we have also $\left\|\phi_{s}\right\|_{\infty} \leq C \delta / \epsilon$, and thus, from (55) and Poincaré inequality we get

$$
\begin{aligned}
& \left(\left\|\rho_{s}\left[\sigma \phi-\chi-(\sigma \varphi-\chi)_{\Omega}\right]\right\|_{2}+2 \sigma\left\|\phi_{s} \rho\right\|_{2}\right)\left\|\rho_{s} \varphi+2 \rho \phi_{s}\right\|_{2} \leq \\
& \frac{\sigma}{2}\left\|\rho_{s} \varphi+2 \rho \phi_{s}\right\|_{2}^{2}+C \delta^{2}\left(\frac{1}{\epsilon^{2}}\|\rho\|_{2}^{2}+\|\nabla \chi\|_{2}^{2}+\delta^{2}\|\nabla \rho\|_{2}^{2}\right) .
\end{aligned}
$$


Finally, by (60) and (61) we have that

$$
\begin{aligned}
\|\nabla \rho\|_{2}^{2}+\frac{1}{2}\|\nabla \chi\|_{2}^{2} & +\frac{\sigma}{2}\left\|\rho_{s} \varphi+2 \rho \phi_{s}\right\|_{2}^{2}+\frac{2}{\epsilon^{2}}\|\rho\|_{2}^{2} \leq C \delta^{2}\left[\frac{1}{\epsilon^{2}}\|\rho\|_{2}^{2}\right. \\
& \left.+\|\nabla \rho\|_{2}^{2}+\|\nabla \chi\|_{2}^{2}+\left\|\rho_{s} \varphi+2 \rho \phi_{s}\right\|_{2}^{2}\right]+\lambda_{r}\left(\left\|\rho_{s} \chi\right\|_{2}^{2}+\|\rho\|_{2}^{2}\right) .
\end{aligned}
$$

For a sufficiently small $\delta$ we obtain that $\lambda_{r} \geq 0$. If $\lambda_{r}=0$ we have, for a sufficiently small value of $\delta$, that

$$
\|\rho\|_{2}^{2}+\|\nabla \chi\|_{2}^{2}=0
$$

Consequently, any eigenfunction associated with any eigenvalue on the imaginary axis (for which $\lambda_{r}=0$ ) must be of the form $v=K i \rho_{s}$ where $K \in \mathbb{R}$ is a constant. From the definition of $D(\mathcal{B})$ we easily conclude that $K=0$. Hence, $\lambda_{r}>0$.

Acknowledgment. This research was supported by US-Israel BSF grant no. 2010194.

\section{References}

[1] S. J. Chapman and D. R. Heron, "A hierarchy of models for superconducting thin films," SIAM J. Appl. Math., vol. 63, no. 6, pp. 2087-2127 (electronic), 2003.

[2] L. P. Gor'kov and G. M. liashberg, "Generalisation of the Ginzburg-Landau equations for non-stationary problems in the case of alloys with paramagnetic impurities," Soviet Phys. J.E.T.P, vol. 27, p. 328, 1968.

[3] Y. Almog, "The interface between the normal state and the fully superconducting state in the presence of an electric current," Commun. Contemp. Math., vol. 14, no. 4, pp. 1250026, 27, 2012.

[4] B. I. Ivlev and N. B. Kopnin, "Electric currents and resistive states in thin superconductors," Advances in Physics, vol. 33, pp. 47-114, 1984.

[5] B. I. Ivlev, N. B. Kopnin, and L. A. Maslova, "Stability of current-carrying states in narrow finite-length superconducting channels," Zh. Eksp. Teor. Fiz., vol. 83, pp. 1533-1545, 1982.

[6] A. J. Dolgert, T. Blum, A. T. Dorsey, and M. Fowler, "Nucleation and growth of the superconducting phase in the presence of a current," Phys. Rev. B, vol. 57, pp. 5432-5443, Mar 1998.

[7] D. Y. Vodolazov, F. M. Peeters, L. Piraux, S. Matefi-Tempfli, and S. Michotte, "Current-voltage characteristics of quasi-one-dimensional superconductors: An s-shaped curve in the constant voltage regime," Physical Review Letters, vol. 91, no. 15, p. 157001, 2003. 
[8] S. Kallush and J. Berger, "Qualitative modifications and new dynamic phases in the phase diagram of one-dimensional superconducting wires driven with electric currents," Phys. Rev. B, vol. 89, p. 214509, Jun 2014.

[9] J. Rubinstein, P. Sternberg, and Q. Ma, "Bifurcation diagram and pattern formation of phase slip centers in superconducting wires driven with electric currents," Physical Review Letters, vol. 99, OCT 192007.

[10] J. Rubinstein and P. Sternberg, "Formation and stability of phase slip centers in nonuniform wires with currents," Physica $C$, vol. 468, pp. 260-263, FEB 15 2008 .

[11] J. Rubinstein, P. Sternberg, and K. Zumbrun, "The Resistive State in a Superconducting Wire: Bifurcation from the Normal State," Archive for Rational Mechanics and Analysis, vol. 195, pp. 117-158, JAN 2010.

[12] J. Rubinstein, P. Sternberg, and J. Kim, "On the behavior of a superconducting wire subjected to a constant voltage difference," SIAM Journal on Applied Mathematics, vol. 70, no. 6, pp. 1739-1760, 2010.

[13] Y. Almog, "The stability of the normal state of superconductors in the presence of electric currents," SIAM Journal on Mathematical Analysis, vol. 40, no. 2, pp. 824-850, 2008.

[14] Q. Du and P. Gray, "High-kappa limits of the time-dependent Ginzburg-Landau model," SIAM J. Appl. Math., vol. 56, no. 4, pp. 1060-1093, 1996.

[15] Q. Du, J. Wei, and C. Zhao, "Vortex solutions of the high- $\kappa$ high-field GinzburgLandau model with an applied current," SIAM J. Math. Anal., vol. 42, no. 6, pp. 2368-2401, 2010.

[16] L. Nirenberg, Topics in nonlinear functional analysis, vol. 6 of Courant Lecture Notes in Mathematics. New York: New York University Courant Institute of Mathematical Sciences, 2001. Chapter 6 by E. Zehnder, Notes by R. A. Artino, Revised reprint of the 1974 original.

[17] S. Agmon, Lectures on elliptic boundary value problems. Prepared for publication by B. Frank Jones, Jr. with the assistance of George W. Batten, Jr. Van Nostrand Mathematical Studies, No. 2, D. Van Nostrand Co., Inc., Princeton, N.J.-Toronto-London, 1965. 